\title{
SOLUTION OF GENERAL INHOMOGENEOUS LINEAR DIFFERENCE EQUATIONS
}

\author{
H. VAN HOA'
}

(Received 19 December 1980; revised 24 February 1982)

\begin{abstract}
The general solution of the $r$ th order inhomogeneous linear difference equation is given in the form

$$
u_{m}=a_{1}^{(n-r)}(n) u_{r-1}+\cdots+a_{r}^{(n-r)}(n) u_{0}+b^{(n-r)}(n) .
$$

The coefficients $a_{r}^{(n-r)}(n), i=2, \ldots, r$, and $b^{(n-r)}(n)$ can be evaluated from $n$ values $a_{1}^{(k)}(n), k=0, \ldots, n-1$, which satisfy an $r$ th order homogeneous linear difference equation. In the $r$ th order homogeneous case and if $n \geqslant 2 r$, the method requires the evaluation of $r$ determinants of successive orders $n-2 r+1, n-2 r+2, \ldots, n-r$. If $r \leqslant n \leqslant 2 r-1$, only $n-r$ determinants are required, with orders varying from 1 to $n-r$. In the second order inhomogeneous case, $a f^{(k)}(n)$ can be evaluated from a continued fraction and a simple product.
\end{abstract}

\section{Introduction}

The $r$ th order homogeneous linear difference equation

$$
\sum_{i=0}^{r} a_{i}(n) u_{n-i}=0, \quad n \geqslant r
$$

where the coefficients $a_{t}(n), i=0,1, \ldots, r$, with $a_{0}(n) \neq 0$, are arbitrary functions of $n$, has been studied by Singh in a recent paper [3]. Singh has given two forms of the solution, namely the matrix form and the determinantal one. The latter, obtained by Cramer's rule, is as follows:

$$
u_{n}=(-1)^{n-r+1} p_{n}^{-1} \sum_{s=1}^{r} D_{s}^{n} u_{r-s}, \quad n \geqslant r
$$

1 School of Mathematics, University of New South Wales, P.O. Box 1, Kensington, N.S.W. 2033. Present address: 39 Norton Street, Ashfield, N.S.W. 2131.

(c) Copyright Australian Mathematical Society 1983 
where

$$
p_{n}=\prod_{l=r}^{n} a_{0}(l)
$$

and

$$
D_{s}^{n}=\left|\begin{array}{ccccccc}
a_{s}(r) & a_{0}(r) & 0 & \cdots & 0 & \cdots & 0 \\
a_{s+1}(r+1) & a_{1}(r+1) & a_{0}(r+1) & \cdots & 0 & \cdots & 0 \\
\vdots & \vdots & \vdots & & \vdots & & \vdots \\
a_{r}(2 r-s) & a_{r-s}(2 r-s) & \vdots & & a_{0}(2 r-s) & \ldots & 0 \\
0 & \vdots & \vdots & \cdots & \vdots & \ldots & 0 \\
\vdots & a_{r}(2 r) & a_{r-1}(2 r) & \cdots & \vdots & \cdots & 0 \\
\vdots & 0 & a_{r}(2 r+1) & \cdots & \vdots & \cdots & 0 \\
\vdots & \vdots & \vdots & & \vdots & & \vdots \\
\vdots & \vdots & \vdots & \cdots & \vdots & \cdots & a_{0}(n-1) \\
\vdots & \vdots & \vdots & \cdots & \vdots & \cdots & a_{1}(n)
\end{array}\right|
$$

The method requires the evaluation of $r$ determinants of order $n-r+1$.

Singh's result generalizes the solution given by Brown [1] in case of the second order homogeneous equation

$$
H(n) u_{n+1}+G(n) u_{n}+F(n) u_{n-1}=0
$$

namely

$$
u_{n+1}=(-1)^{n}\left\{u_{0} F(1) C_{2}^{n}+u_{1} C_{1}^{n}\right\} / \prod_{s=1}^{n} H(s),
$$

where $C_{1}^{n}$ and $C_{2}^{n}$ are tridiagonal determinants of orders $n$ and $n-1$ defined by

$$
C_{m}^{n}=\left|\begin{array}{ccccccccc}
G(m) & H(m) & 0 & \ldots & . & \ldots & \ldots & \ldots & 0 \\
F(m+1) & G(m+1) & H(m+1) & 0 & \ldots & \ldots & . & \ldots & 0 \\
0 & F(m+2) & G(m+2) & H(m+2) & 0 & \ldots & \ldots & . & 0 \\
\vdots & . & \ldots & \ldots & \ldots & \ldots & \ldots & . & 0 \\
0 & \ldots & \ldots & . & \ldots & F(n-2) & G(n-2) & H(n-2) & 0 \\
\vdots & \ldots & \ldots & \ldots & \ldots & 0 & F(n-1) & G(n-1) & H(n-1) \\
0 & \ldots & \ldots & \ldots & & \ldots & 0 & F(n) & G(n)
\end{array}\right| .
$$


For the case (1.4), in a recent paper, Love [2] has proposed an alternative method for evaluating the solution from a continued fraction and a simple product, as follows:

$$
u_{n+1}=u_{0} \prod_{s=0}^{n} p_{s}
$$

where

$$
\begin{aligned}
& p_{s}=\frac{u_{s+1}}{u_{s}}=-\frac{G(s)}{H(s)}-\frac{\frac{F(s)}{H(s)}}{-\frac{G(s-1)}{H(s-1)}-\frac{F(s-1)}{H(s-1)}} \\
& -\frac{G(s-2)}{H(s-2)}-\cdots \\
& \ldots-\frac{F(1)}{\frac{H(1)}{u_{0}}}
\end{aligned}
$$

Love's method does not apply to the general case of (1.1), nor to the second order inhomogeneous linear equation.

In the following section, we consider the general $r$ th order inhomogeneous linear difference equation reduced to the form

$$
u_{n}=\sum_{i=1}^{r} a_{i}(n) u_{n-i}+b(n), \quad n \geqslant r
$$

where $a_{t}(n), i=1,2, \ldots, r$, are arbitrary functions of $n$, and $a_{r}(n) \neq 0$.

In the homogeneous case, $b(n) \equiv 0$, our method contributes another approach to the problem, different from Singh's. Moreover, if $n \geqslant 2 r$, instead of $r$ determinants of order $n-r+1$ as in Singh's method, our approach requires $r$ determinants of successive orders $n-2 r+1, n-2 r+2, \ldots, n-r$. If $r \leqslant n \leqslant 2 r-1$, only $n-r$ determinants are required, with orders varying from 1 to $n-r$.

Finally, in case of the second order inhomogeneous linear equations, our results make Love's method applicable. 


\section{Method of solution}

Assume that $n>r$. In (1.9), writing $n-1$ for $n$ and eliminating $u_{n-1}$ between the obtained equation and (1.9), we have

$$
u_{n}=\sum_{i=1}^{r} a_{i}^{(\mathrm{l})}(n) u_{n-i-1}+b^{(\mathrm{l})}(n), \quad n>r
$$

where

$$
a_{i}^{(1)}(n)=a_{i}(n-1) a_{1}(n)+a_{i+1}(n), \quad i=1, \ldots, r,
$$

and

$$
b^{(1)}(n)=b(n-1) a_{1}(n)+b(n),
$$

with the convention $a_{i}(n) \equiv 0$ if $i>r$. Repeating the above operation $n-r$ times by taking for $n$ in (1.9) successive values $n-1, n-2, \ldots, r$, we obtain

$$
u_{n}=\sum_{i=1}^{r} a_{i}^{(n-r)}(n) u_{r-i}+b^{(n-r)}(n) .
$$

The coefficients $a_{i}^{(n-r)}(n)$ and $b^{(n-r)}(n)$ satisfy the formulae

$$
\begin{aligned}
& a_{1}^{(k)}(n)=a_{i}(n-k) a_{1}^{(k-1)}(n)+a_{i+1}^{(k-1)}(n), \\
& b^{(k)}(n)=b(n-k) a_{1}^{(k-1)}(n)+b^{(k-1)}(n),
\end{aligned}
$$

where $1 \leqslant k \leqslant n-r ; i=1, \ldots, r$ and with the conventions

$$
\begin{aligned}
a_{i}^{(0)}(n) & \equiv a_{t}(n), \quad i=1, \ldots, r, \\
b^{(0)}(n) & \equiv b(n), \\
a_{i}^{(k)}(n) & \equiv 0 \quad \text { for all } k \quad \text { if } i>r \text { or } i<1 .
\end{aligned}
$$

It is easy to see that the problem can be reduced to the evaluation of $n$ coefficients $a_{1}^{(k)}(n)$, where $k$ varies from 0 to $n-1$.

Indeed, equation (2.5) can be written as follows

$$
a_{i}^{(k)}(n)=-a_{i-1}(n-k-1) a_{1}^{(k)}(n)+a_{i-1}^{(k+1)}(n) .
$$

By performing backward iterations with respect to $i$ and down to $i=1$ and by noting the conventions (2.9) and $a_{0}(n) \equiv-1$, we can obtain

$$
a_{i}^{(k)}(n)=-\sum_{j=1}^{i} a_{i-j}(n-k-j) a_{1}^{(k+j-1)}(n) .
$$

Similarly, we can deduce from (2.6) the relation

$$
b^{(k)}(n)=b(n)+\sum_{j=1}^{k} b(n-k+j-1) a_{1}^{(k-j)}(n) .
$$


In the case of the homogeneous equation, the number of necessary coefficients $a_{1}^{(k)}(n)$ reduces to $r$ when $k$ varies from $n-r$ to $n-1$.

On the other hand, by performing forward iterations of equation (2.5) with respect to $i$ and up to $i=r$ and by making use of the condition (2.9), we can obtain the relation

$$
a_{i}^{(k)}(n)=\sum_{j=1}^{r-1+1} a_{i+j-1}(n-k+j-1) a_{1}^{(k-j)}(n), \quad i \leqslant r \leqslant k+i-1 \text {, }
$$

which gives the following difference equation for $a_{1}^{(k)}(n)$ when $i=1$ :

$$
a_{1}^{(k)}(n)=\sum_{j=1}^{r} a_{j}(n-k+j-1) a_{1}^{(k-\jmath)}(n), \quad k \geqslant r \geqslant 1 .
$$

It is surprising that this equation is similar to the homogeneous part of the original equation (1.9), with modified coefficients.

The general solution $a_{1}^{(k)}(n)$ can be evaluated as functions of the first $r$ values,

$$
\begin{aligned}
a_{1}^{(0)}(n)= & a_{1}(n), \\
a_{1}^{(1)}(n)= & a_{1}(n-1) a_{1}(n)+a_{2}(n), \\
a_{1}^{(2)}(n)= & a_{1}(n-2) a_{1}(n-1) a_{1}(n) \\
& \quad+a_{1}(n-2) a_{2}(n)+a_{1}(n) a_{2}(n-1)+a_{3}(n), \\
\ldots \cdots \cdots \cdots \cdots \cdots \cdots \cdots \cdots & \cdots \cdots \cdots \cdots \cdots \\
a_{1}^{(r-1)}(n)= & a_{1}(n-r+1) a_{1}^{(r-2)}(n)+a_{2}^{(r-2)}(n) .
\end{aligned}
$$

Assume that $k \geqslant r$. Letting $k=k, k-1, \ldots, r$ in (2.14), we obtain a system of $k-r+1$ equations which effectively gives $a_{1}^{(k)}(n)$ in determinantal form.

We distinguish 2 cases:

Case $1: r \leqslant k \leqslant 2 r-1$.

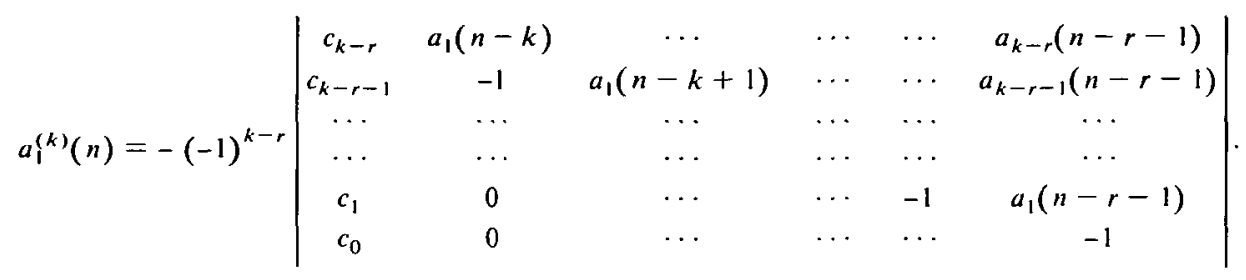


Case 2: $k \geqslant 2 r$.

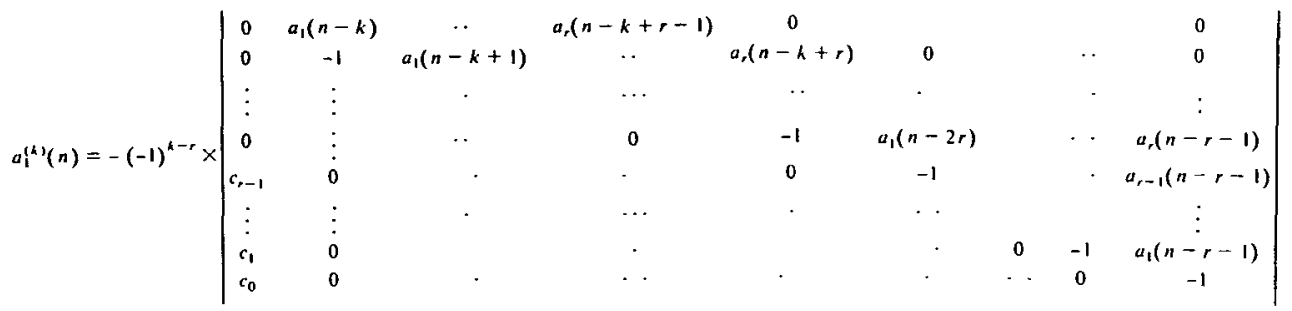

In (2.16) and (2.17), the quantities $c_{m}$ are defined as

$$
c_{m}=-\sum_{j=m+1}^{r} a_{j}(n-r-m+j-1) a_{1}^{(r+m-j)}(n), \quad 0 \leqslant m \leqslant r-1 .
$$

In the $r$ th order inhomogeneous case, the solution requires the evaluation of $n-r$ determinants like (2.16) or (2.17) of orders $1,2, \ldots$, and $n-r$, corresponding to $k=r, r+1, \ldots, n-1$. In the $r$ th order homogeneous case, the number of determinants to be evaluated remains the same if $r \leqslant n \leqslant 2 r-1$ (in this case, $n-r<r$ ). If $n \geqslant 2 r$, we have to evaluate $r$ determinants of orders $n-2 r+1$, $n-2 r+2, \ldots, n-r$ corresponding to $k=n-r, \ldots, n-1$. In both cases, it is clear that the proposed method reduces either the number or the orders of the determinants to be evaluated in comparison with Singh's method. In the case of the second order homogeneous equation, our results require the evaluation of 2 determinants of orders $n-2$ and $n-3$ (assume that $n \geqslant 4$ ) instead of $n-1$ and $n-2$ as in Brown's method.

\section{The second order inhomogeneous case and Love's method}

Love's method does not apply to the second order inhomogeneous equation

$$
u_{n}=a_{1}(n) u_{n-1}+a_{2}(n) u_{n-2}+b(n), \quad n \geqslant 2 .
$$

In this case, the general solution is given by (2.4) with $r=2$,

$$
u_{n}=a_{1}^{(n-2)}(n) u_{1}+a_{2}^{(n-2)}(n) u_{0}+b^{(n-2)}(n),
$$

where

$$
a_{2}^{(n-2)}(n)=-a_{1}(1) a_{1}^{(n-2)}(n)+a_{1}^{(n-1)}(n),
$$




$$
b^{(n-2)}(n)=b(n)+\sum_{j=1}^{n-2} b(j+1) a_{i}^{(n-J-2)}(n) .
$$

The coefficients $a_{1}^{(k)}(n)$ can now be evaluated by Love's method. Indeed, they satisfy the second order homogeneous linear difference equation

$$
a_{1}^{(k)}(n)=a_{1}(n-k) a_{1}^{(k-1)}(n)+a_{2}(n-k+1) a_{1}^{(k-2)}(n),
$$

which can bc reâriangể às

$$
\frac{a_{1}^{(k)}(n)}{a_{1}^{(k-1)}(n)}=a_{1}(n-k)+\frac{a_{2}(n-k+1)}{\left[a_{1}^{(k-1)}(n) / a_{1}^{(k-2)}(n)\right]} .
$$

Therefore, the ratios

$$
p^{(k)}=\frac{a_{1}^{(k)}(n)}{a_{1}^{(k-1)}(n)}
$$

can be evaluated in continued fraction form

$$
\begin{gathered}
p^{(k)=} a_{1}(n-k)+\frac{a_{2}(n-k+1)}{a_{1}(n-k+1)+a_{1}(n-k+2)+} \frac{a_{2}(n-k+2)}{a_{1}} \cdots \\
\cdots \frac{a_{2}(n-2)}{a_{1}(n-2)+\frac{a_{2}(n-1) a_{1}(n)}{a_{1}(n-1) a_{1}(n)+a_{2}(n)} .}
\end{gathered}
$$

Finally, $a_{1}^{(k)}(n)$ can be expressed as a product of ratios of successive $a_{1}^{(s)}(n)$ :

$$
\begin{aligned}
a_{1}^{(k)}(n) & =\frac{a_{1}^{(k)}}{a_{1}^{(k-1)}} \frac{a_{1}^{(k-1)}}{a_{1}^{(k-2)}} \cdots \frac{a_{1}^{(1)}}{a_{1}^{(0)}} a_{1}^{(0)} \\
& =a_{1}(n) \prod_{s=1}^{k} p^{(s)} .
\end{aligned}
$$

\section{Acknowledgements}

The author wishes to thank the referees for their helpful suggestions and comments.

\section{References}

[1] A. Brown, "Solution of a linear difference equation", Bull. Austral. Math. Soc. 11 (1974), 325-331.

[2] J. D. Love, "Solution of homogeneous linear difference equations", J. Austral. Math. Soc. Ser. B 21 (1980), 293-296.

[3] V. N. Singh, "Solution of a general homogeneous linear difference equation", J. Austral. Math. Soc. Ser. B 22 (1980), 53-57. 